\title{
The forgotten role of adjuvant immune checkpoint inhibitors in preventing melanoma brain metastasis
}

\author{
Elie El Rassy*,1, Fadi Farhat ${ }^{1} \&$ Joseph Kattan $^{1}$ \\ ${ }^{1}$ Department of Medical Oncology, Hotel Dieu de France University Hospital, Faculty of Medicine, Saint Joseph University, Lebanon \\ *Author for correspondence: elie.rassy@hotmail.com
}
"Although the available evidence for $\mathrm{ICl}$ activity in preventing CNS metastasis is very limited, its implication in early disease is high relevant to clinical practice given the natural history of melanoma with CNS metastasis. The $\mathrm{ICl}$ adjuvant regimens have shown clinically meaningful reduction in the risk of melanoma recurrence"

First draft submitted: 26 July 2018; Accepted for publication: 17 October 2018; Published online: 23 November 2018

Keywords: adjuvant $\bullet$ brain metastases $\bullet$ immune checkpoint inhibitors $\bullet$ intracranial $\bullet$ ipilimumab $\bullet$ melanoma $\bullet$ nivolumab • pembrolizumab

Localized melanoma is commonly cured with simple excision except for high-risk stage III melanoma that is often complicated with distant metastasis and death. A significant portion of these relapses develop in the CNS, which results in a shortened survival and altered quality of life [1]. Initial attempts to prevent the metastatic spread to the CNS relied on the administration of adjuvant therapies following resection [1]. Unfortunately, these attempts failed and active efforts were needed to identify more efficacious adjuvant regimens. Recently, the immune checkpoint inhibitors (ICI) showed high activity in metastatic melanoma, which constituted the backdrop for its evaluation in the adjuvant setting [2-4]. The dilemma remains whether ICI would prevent CNS relapse in resected high-risk stage III melanoma.

Melanoma tends to metastasize in the CNS according to the 'seed and soil' theory with a specific interaction between the melanoma cells and the CNS (Figure 1). A CNS metastasis-initiating melanoma cell escapes the immune surveillance during intravasation, adhesion to endothelial cells, extravasation into the parenchyma, vascular cooption, angiogenesis and proliferation [5]. The interaction of the melanoma cells with the soil microenvironment after extravasation seems the rate limiting step of the process. The immune system at this level may promote or hamper metastasis within the perivascular niche by switching between M1 (tumor-suppressive) and M2 (tumor-tolerant) phenotype [6]. The M2 component of the immune system alters the adaptive immunity through immunosuppressive factors such as PD-L1 and PD-1 [7]. Thus metastasis may not soil the CNS either due to the failure of solitary melanoma cells to initiate growth or early micrometastases to continue growth into macroscopic tumors [8].

As preclinical models have shown that ICI activate T-cells trafficking across the blood-brain barrier into the perivascular niche, adjuvant ICI may be protective of CNS metastasis in completely resected high-risk melanoma that has already circulating tumor cells. To date, three adjuvant ICI trials have enrolled 1895 patients with resected stage III melanoma (Supplementary Table 1). These patients were characterized by BRAF mutations in 41.3-47.7\%, macroscopic lymph node involvement in 33.9-58.8\% and ulceration of the primary tumor in $36.9-41.5 \%$. After a median follow-up of 1.25-2.74 years, the rate of 1 -year relapse-free survival was $60.8-75.4 \%$. Unfortunately, none of these trials reported on the pattern of CNS relapse following adjuvant ICI [2-4].

The activity of ICI in CNS metastasis has not been recognized until recently $[9,10]$. The pivotal ICI trials in metastatic melanoma did not report the intracranial response rates probably due to the small numbers of patients with CNS metastasis (3-10\%) [11-13]. One study evaluating the combination of nivolumab plus ipilimumab in metastatic melanoma reported the lowest rate of CNS progression in comparison to nivolumab and ipilimumab (nivolumab plus ipilimumab 11\%, nivolumab 17\% and ipilimumab 14\%) [14]. A recently published Phase II 


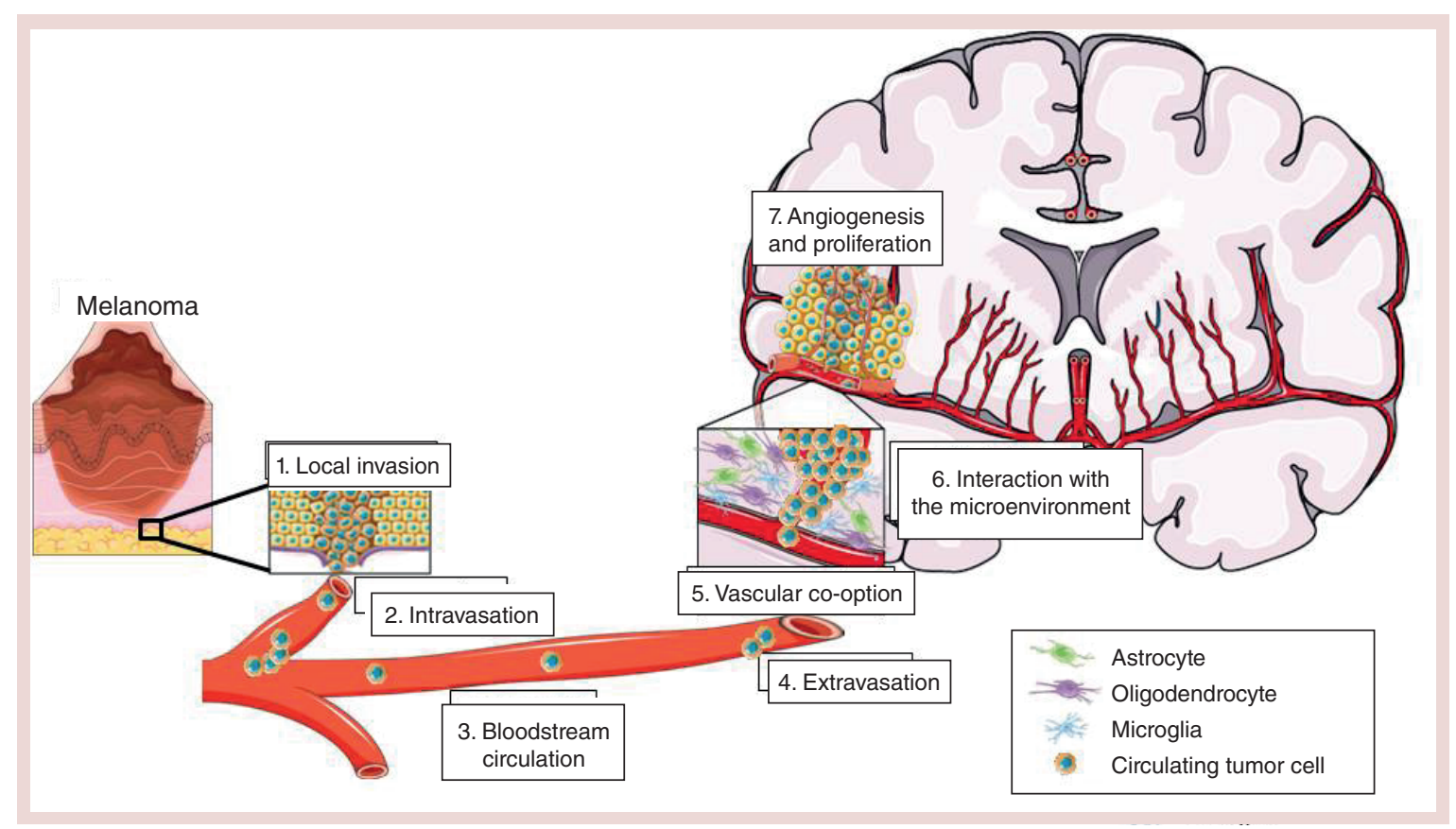

Figure 1. The successive 'seed and soil' steps in the development of central nervous metastasis in melanoma. Reproduced with permission from http://smart.servier/com.

trial (NCT02374242) reported on the safety and efficacy of nivolumab plus ipilimumab in melanoma patients with CNS metastasis [15]. The intracranial response rate in melanoma patients with untreated asymptomatic CNS metastases was $46 \%$ ( 14 of 35 patients) with nivolumab plus ipilimumab and $20 \%$ ( 5 of 25 patients) with nivolumab monotherapy [15]. Another Phase II trial, NCT02320058, assessed the intracranial response rate of the combination nivolumab plus ipilimumab in metastatic melanoma with nonirradiated brain metastasis (tumor diameter between 0.5 and $3 \mathrm{~cm}$ and no neurologic symptoms) [16]. Among the 94 patients eligible for this study, the intracranial response rate was $57 \%$ and treatment-related grade $3 / 4$ adverse events rates was $55 \%$ (CNS events in 7\%). The safety profile of nivolumab plus ipilimumab was similar among patients with or without CNS metastasis [16]. Interestingly, the combination of nivolumab plus ipilimumab prevented intracranial progression for $>6$ months in $64 \%$ of patients [16]. Pembrolizumab has also shown promising intracranial response rates of $22 \%$ ( 4 of 18 patients) in small asymptomatic CNS metastasis [17].

Although the available evidence for ICI activity in preventing CNS metastasis is very limited, its implication in early disease is high relevant to clinical practice given the natural history of melanoma with CNS metastasis. The ICI adjuvant regimens have shown clinically meaningful reduction in the risk of melanoma recurrence but did not report on the occurrence of CNS metastasis at recurrence [18]. Similarly, targeted therapy adjuvant regimens present the same issue although it presents an intracranial response rate of $58 \%$ among melanoma patients with BRAF V600 mutation and asymptomatic brain metastases $[19,20]$. This achieves similar intracranial response rates to nivolumab plus ipilimumab; however, the duration of intracranial response was shorter with the combination of dabrafenib plus trametinib (6.5 months vs not reached after median follow-up of 14 months) [15,16,20].

The longer duration of systemic control for ICI compared with targeted therapy and the higher intracranial responses with combination ICI compared with monotherapy ICI lead us to speculate that adjuvant ICI combination therapy seems the best option to reduce the risk of CNS metastasis in high-risk melanoma patients. The main concern remains in identifying the risk factors for CNS metastasis in melanoma to avoid overtreatment and excessive toxicities. Herein raises the need to enhance the understanding of the patient and tumor-related factors that may increase the risk of CNS metastasis. These factors may be considered to build risk models that would stratify the risk of CNS metastasis thus the necessity for aggressive adjuvant treatment with high intracranial activity. Such data may be collected from the adjuvant trials of ICI in high-risk melanoma. 


\section{Financial \& competing interests disclosure}

The authors have no relevant affiliations or financial involvement with any organization or entity with a financial interest in or financial conflict with the subject matter or materials discussed in the manuscript. This includes employment, consultancies, honoraria, stock ownership or options, expert testimony, grants or patents received or pending, or royalties.

No writing assistance was utilized in the production of this manuscript.

\section{Supplementary data}

To view the supplementary data that accompany this article visit the journal website at: www.futuremedicine.com/doi/suppl/10. 2217/imt-2018-0108

\section{References}

1. Samlowski WE, Moon J, Witter M et al. High frequency of brain metastases after adjuvant therapy for high-risk melanoma. Cancer Med. 6(11), 2576-2585 (2017).

2. Eggermont AMM, Chiarion-Sileni V, Grob J-J et al. Adjuvant ipilimumab versus placebo after complete resection of high-risk stage III melanoma (EORTC 18071): a randomised, double-blind, Phase III trial. Lancet Oncol. 16(5), 522-530 (2015).

3. Eggermont AMM, Blank CU, Mandala M et al. Adjuvant pembrolizumab versus placebo in resected stage III melanoma. N. Engl. J. Med. 378(19), 1789-1801 (2018).

4. Weber J, Mandala M, Del Vecchio M et al. Adjuvant nivolumab versus ipilimumab in resected stage III or IV melanoma. $N$. Engl. J. Med. 377(19), 1824-1835 (2017).

5. Fidler IJ, Yano S, Zhang R-D, Fujimaki T, Bucana CD. The seed and soil hypothesis: vascularisation and brain metastases. Lancet Oncol. 3(1), 53-57 (2002).

6. Owyong M, Hosseini-Nassab N, Efe G et al. Cancer immunotherapy getting brainy: visualizing the distinctive CNS metastatic niche to illuminate therapeutic resistance. Drug Resist. Updat. 33-35, 23-35 (2017).

7. Berghoff AS, Fuchs E, Ricken G et al. Density of tumor-infiltrating lymphocytes correlates with extent of brain edema and overall survival time in patients with brain metastases. Oncoimmunology 5(1), e1057388 (2016).

8. Luzzi KJ, MacDonald IC, Schmidt EE et al. Multistep nature of metastatic inefficiency: dormancy of solitary cells after successful extravasation and limited survival of early micrometastases. Am. J. Pathol. 153(3), 865-873 (1998).

9. Rassy EE, Botticella A, Kattan J, Péchoux CL, Besse B, Hendriks L. Non-small cell lung cancer brain metastases and the immune system: from brain metastases development to treatment. Cancer Treat. Rev. 0(0) (2018). www.cancertreatmentreviews.com/article/S0305-7372(18)30092-6/fulltext

10. Kattan J, Rassy EE, Assi T, Bakouny Z, Pavlidis N. A comprehensive review of the role of immune checkpoint inhibitors in brain metastasis of renal cell carcinoma origin. Crit. Rev. Oncol. Hematol. 130, 60-69 (2018).

11. Schachter J, Ribas A, Long GV et al. Pembrolizumab versus ipilimumab for advanced melanoma: final overall survival results of a multicentre, randomised, open-label Phase III study (KEYNOTE-006). Lancet Lond. Engl. 390(10105), 1853-1862 (2017).

12. Hodi FS, O’Day SJ, McDermott DF et al. Improved survival with ipilimumab in patients with metastatic melanoma. $N$. Engl. J. Med. 363(8), 711-723 (2010).

13. Robert C, Long GV, Brady B et al. Nivolumab in previously untreated melanoma without BRAF mutation. N. Engl. J. Med. 372(4), $320-330$ (2015).

14. Wolchok JD, Kluger H, Callahan MK et al. Nivolumab plus Ipilimumab in advanced melanoma. N. Engl. J. Med. 369(2), 122-133 (2013).

15. Long GV, Atkinson V, Lo S et al. Combination nivolumab and ipilimumab or nivolumab alone in melanoma brain metastases: a multicentre randomised Phase II study. Lancet Oncol. 19(5), 672-681 (2018).

16. Tawbi HA, Forsyth PA, Algazi A et al. Combined nivolumab and ipilimumab in melanoma metastatic to the brain. N. Engl. J. Med. 379(8), 722-730 (2018).

17. Goldberg SB, Gettinger SN, Mahajan A et al. Pembrolizumab for patients with melanoma or non-small-cell lung cancer and untreated brain metastases: early analysis of a non-randomised, open-label, Phase II trial. Lancet Oncol. 17(7), 976-983 (2016).

18. Schuchter LM. Adjuvant melanoma therapy - head-spinning progress. N. Engl. J. Med. 377(19), 1888-1890 (2017).

19. Long GV, Hauschild A, Santinami M et al. Adjuvant dabrafenib plus trametinib in stage III BRAF-mutated melanoma. N. Engl. J. Med. 377(19), 1813-1823 (2017).

20. Davies MA, Saiag P, Robert C et al. Dabrafenib plus trametinib in patients with BRAFV600-mutant melanoma brain metastases (COMBI-MB): a multicentre, multicohort, open-label, Phase II trial. Lancet Oncol. 18(7), 863-873 (2017). 
